\title{
Is there a role for sentinel lymph node biopsy in the management of sarcoma?
}

\author{
Dan G. Blazer III, Michael S. Sabel*, Vernon K. Sondak \\ Department of General Surgery, Division of Surgical Oncology, University of Michigan, 3304 Cancer Center, \\ 1500 East Medical Center Drive, Ann Arbor, MI 48109, USA
}

\begin{abstract}
Is there a role for sentinel lymph node (SLN) biopsy in the management of sarcoma? Sentinel node biopsy has dramatically changed the management of melanoma and breast cancer, helping surgeons avoid radical lymphadenectomies in node negative patients who would previously have undergone a more morbid operation with little benefit, or remained pathologically unstaged. Many investigators have explored the use of lymphatic mapping for malignancies other than breast cancer or melanoma. Lymphatic mapping and sentinel node biopsy has not been investigated in the management of sarcomas, which is not surprising given that the majority of sarcomas spread by local extension or hematogenously. Regional lymph node metastases are rare; developing in about $3-10 \%$ of patients with localized disease. However, among certain subtypes of high-grade sarcomas there is a propensity for regional lymph node metastases. These include rhabdomyosarcoma, epithelioid sarcoma, clear cell sarcoma, synovial sarcoma, and vascular sarcomas. It is in these particular subtypes that there may be a benefit to SLN biopsy.
\end{abstract}

(C) 2003 Elsevier Ltd. All rights reserved.

Keywords: Sarcoma; Sentinel lymph node biopsy

\section{Introduction}

The use of sentinel lymph node (SLN) biopsy to assess the regional lymphatic basin for the presence of metastatic disease is quickly becoming the standard of care in the management of melanoma and breast cancer. The concept of lymphatic mapping and SLN biopsy was first described by Cabanas in 1977 in regards to penile cancer [1]. The concept was further developed by Morton, who reported its use for melanoma in 1992 [2]. The core concept behind the sentinel node is that the primary tumor reliably drains to one or a few lymph nodes in the regional lymphatic basin, and therefore histopathologic analysis of these "sentinel" nodes reflects the disease status of the entire lymphatic field. Several studies have supported this hypothesis in both melanoma [3-5] and breast cancer [6-8]. In utilizing SLN biopsy to stage the regional lymphatic basin, surgeons hope to avoid radical lymphadenectomies node negative patients who would previously have undergone

\footnotetext{
*Corresponding author. Tel.: + 1-734-936-5827; fax: + 1-734-947-9647.

E-mail address: msabel@umich.edu (M.S. Sabel).
}

a more morbid operation with little benefit, or remained pathologically unstaged.

Given the potential advantages of SLN biopsy, many investigators have explored the use of lymphatic mapping for malignancies other than breast cancer or melanoma. These include lung cancer [9], colon cancer $[10,11]$, squamous cell carcinoma of the head and neck [12,13], gynecologic cancers [14-16], thyroid cancer $[17,18]$, Merkel cell carcinoma [19-21], upper gastrointestinal cancers $[22,23]$ and non-small cell lung cancer [24]. Though its role has yet to be defined, surgeons are hoping this minimally invasive technique may serve as a means to improve staging, better predict prognosis and utilize adjuvant therapies and decrease morbidity by avoiding unnecessary major lymphadenectomies.

One malignancy for which SLN biopsy has not been investigated is sarcoma. That the technique of SLN biopsy has not been investigated in the management of sarcomas is not particularly surprising given that the majority of sarcomas spread by local extension or hematogenously. Regional lymph node metastases are rare; developing in about $3-10 \%$ of patients with localized disease [25]. Recurrence in the regional nodal basin is also rare, representing $4-10 \%$ of recurrences, 
often with distant metastases as well [26,27]. Classic teaching in sarcoma stresses radical excision of the primary without need for lymph node evaluation or lymphadenectomy. Though the overall incidence of regional lymph node metastases in sarcoma is low, there do appear to be certain histologic subtypes in which there is an increased incidence. Skinner and Eilber reviewed a number of different studies demonstrating the increased incidence of regional lymph node metastases in certain types of sarcomas [25]. They concluded that lymph node metastases occurred almost exclusively in high-grade lesions. Among high-grade sarcomas, only certain subtypes such as rhabdomyosarcoma, epithelioid sarcoma, clear cell sarcoma, synovial sarcoma, and vascular sarcomas appeared to have a propensity for regional lymph node metastatic spread. It is in these particular subtypes that benefits of SLN biopsy might be realized. In this mini-review for Surgical Oncology, we present an argument for the selective use of SLN biopsy in the management of sarcoma.

\section{Rhabdomyosarcoma}

Rhabdomyosarcoma, sarcomas with demonstrated skeletal muscle differentiation, are common in children and rare in adults. Rhabdomyosarcoma demonstrated regional lymph node metastasis in $11-36 \%$ of cases [25,28-30]. As such, it is clearly one of the histologic types of sarcoma with a higher than average likelihood of regional nodal involvement. It is also the only histologic type of sarcoma for which regional nodal treatment is commonly considered as part of initial therapy.

\subsection{Pediatric rhabdomyosarcoma}

SLN biopsy is likely to ultimately prove to be beneficial in the initial management of pediatric rhabdomyosarcoma. Rhabdomyosarcoma is the most common soft tissue sarcoma in children under 15 years of age and is the sixth most common form of cancer in childhood. Pediatric rhabdomyosarcoma is clearly a responsive tumor, with defined roles for adjuvant therapy with radiation and systemic chemotherapy. There are several sites where these tumors preferentially occur. Rhabdomyosarcomas originating in the extremities and the genitourinary tract appear to be particularly associated with regional lymphatic spread. One review found that the percentage of patients with nodal metastases from extremity lesions was $12 \%$ [31]. The presence or absence of regional lymphatic spread in extremity lesions was a significant prognostic factor in 3year survival rates in these patients. A follow-up study confirmed the necessity of surgically evaluating lymph node basins in extremity rhabdomyosarcoma in order to more accurately stage patients, with the ultimate hope of using improved staging to develop optimal risk-based therapies [32]. Performing SLN biopsy in these children may assist oncologists in projecting survival for these children while at the same time preventing unnecessary major lymphadenectomies. Those with positive SLNs could undergo completion lymphadenectomy, and be treated systemically as for clinically node-positive disease.

In the case of rhabdomyosarcoma of the genitourinary tract in children, regional lymphatic spread has been reported in up to $24 \%$ of children with this type of disease [31]. As with pediatric rhabdomyosarcoma of the extremities, understanding the nodal status in these patients directly impacts therapy. Patients with known nodal disease of the regional lymphatic basin in rhabdomyosarcomas of the bladder, prostate, vagina/ uterus, and paratesticular tissues routinely undergo systemic chemotherapy and radiation therapy to the regional lymphatic basin [33].

The evolving management of paratesticular rhabdomyosarcoma over the past 2 decades is especially illustrative of the potential benefit of SLN biopsy in the management of pediatric genitourinary rhabdomyosarcoma. Initially, all patients with paratesticular rhabdomyosarcoma required ipsilateral retroperitoneal lymph node dissection. Subsequently, the Intergroup Rhabdomyosarcoma Study Group recommended clinical evaluation of retroperitoneal lymph nodes using computerized tomography [34]. Only those patients with suspicious lymph node involvement on CT scan underwent surgical evaluation of the lymph node basin. Comparison of these two approaches suggested that reliance on CT scan evaluation of the regional lymph node basin resulted in understaging of lymphatic spread and resulted in underutilization of effective adjuvant therapy [34]. Developing SLN protocols seems a very appealing technology as a means to avoid the morbidity of retroperitoneal lymph node dissection in those patients with clinically localized disease.

Of course, techniques for identifying sentinel nodes from genitourinary tract primaries are far less developed than those for tumors arising on the extremities and trunk. Given the focus of pediatric oncologists on treating rhabdomyosarcoma patients in nationwide clinical trials, prospective evaluation of SLN biopsy in this disease seems feasible and worthwhile, but may require new approaches not routinely used when SLN biopsy is performed for other tumor types.

\subsection{Adult rhabdomyosarcoma}

In contrast to the pediatric population, the literature describing adult rhabdomyosarcoma is limited, reflecting its rarity and the paucity of prospective trials in adult sarcomas. The prognosis for adults diagnosed with 
rhabdomyosarcoma is poor-worse than pediatric sarcomas and worse than other adult soft tissue sarcomas of comparable stage and grade. In one series, the overall 5- and 10-year survival rate for patients diagnosed with rhabdomyosarcoma was $31 \%$ and $27 \%$, respectively [35]. Relatively little is known about the frequency and prognostic impact of nodal status in adult patients treated with multimodality therapy. Hence, while SLN biopsy in adult rhabdomyosarcoma appears to merit prospective evaluation, its overall impact on the disease is likely to be small at best.

\section{Epithelioid sarcoma}

Epithelioid sarcoma is a rare high grade soft tissue sarcoma that presents most commonly in adult males in their 20s and 30s and occurs almost exclusively in the extremities. Because of an innocuous presentation, diagnosis is often delayed. Five- and ten-year overall survival is $70 \%$ and $42 \%$, respectively [36]. Epithelioid sarcoma clearly has a propensity for regional lymphatic spread [37]. Regional lymph node metastases in these patients ranges from $17 \%$ to $80 \%$ [25,28-30]. A more recent report demonstrated regional lymph node metastases during the course of the disease to be $44 \%$ [38]. Lymphatic spread has been shown to be an independent prognostic factor in predicting survival rates for these patients [39].

With conservative surgery, nodal failure rate ranges between $19 \%$ and $44 \%$ [38,40,41]. However, nodal failures are often associated with distant metastases, suggesting that nodal failures represent a component of widespread dissemination rather than a purely locoregional event. Routine treatment of the nodal basin, either by surgery or irradiation, appears unlikely to benefit these patients $[38,42]$. With such a high incidence of regional spread though, evaluation of the regional lymph node basin by lymphatic mapping and SLN biopsy may be an excellent strategy for selecting those patients who might benefit from lymphadenectomy or early systemic therapy [40].

\section{Clear cell sarcoma}

Clear cell sarcoma, often referred to as malignant melanoma of the soft parts, has a distinct behavior from other sarcomas - including a tendency to nodal recurrence and widespread metastasis throughout the body. In its pattern of spread, it more closely resembles melanoma than sarcoma, hence the alternate name melanoma of soft parts. It is a rare tumor constituting $1 \%$ of all soft tissue sarcomas, with only about 300 cases having been described in the literature to date [43]. It arises most commonly in the extremities as a bulky, locally invasive tumor. As such, it is a logical candidate for SLN biopsy.

The incidence of lymph node metastases in clear cell sarcoma range from $25 \%$ to $50 \%[25,43]$. Complete surgical excision is the standard of care in the management of clear cell sarcoma. The role of adjuvant chemotherapy and radiation therapy has yet to be determined. At this time, tumor size has been the only feature to predict tumor recurrence, with tumors greater than $5 \mathrm{~cm}$ demonstrating a high incidence of metastatic spread. Currently, those patients with tumor size $>5 \mathrm{~cm}$ undergo some form of adjuvant therapy, primarily doxorubicin-based chemotherapy [43]. However, because this sarcoma is so rare, it has been difficult to sort out optimal clinical management. With 5-, 10-, and 20 -year survival of $67 \%, 33 \%$, and less than $10 \%$, respectively, there is work to be done to better manage this rare sarcoma [43]. The addition of SLN biopsy to the management of clear cell sarcoma could be a valuable technique to better predict prognosis and to further refine adjuvant therapy. Equally important will be ongoing biologic studies to better define the origin and nature of this rare tumor, in order to determine if it should be treated like a sarcoma or a melanoma [44].

\section{Synovial sarcoma}

Synovial sarcomas account for 7-8\% of all soft tissue sarcomas and are the most common non-rhabdomyosarcoma soft tissue sarcomas in pediatric patients. Overall 5-year survival rates for patients diagnosed with synovial sarcoma ranges from $57 \%$ to $88 \%$ [45,46]. Review of the literature demonstrates regional lymphatic spread between $2 \%$ and $17 \%$ of the time [25,28-30]. As such, synovial sarcoma is the most common form of sarcoma for which regional spread is a potential clinical concern. More than $90 \%$ of these sarcomas are found in the extremities or trunk. In one recent report on synovial sarcoma, the authors contend that complete resection with clear margins and evaluation of regional lymphatic spread is the treatment of choice for this tumor [47]. The role of radiation and chemotherapy continues to evolve in the management of these sarcomas, but synovial sarcomas also appear to be among the most responsive of adult sarcomas to chemotherapy [48]. The addition of SLN evaluation in the management of synovial sarcoma could possibly refine the role of chemoradiation protocols in the future, particularly if subgroups of synovial sarcoma with higher rates of nodal spread could be identified. Given the variable reports about the true incidence of regional spread in synovial sarcoma, any evaluation of sentinel node biopsy in this disease should clearly be done in the context of a prospective trial. 


\section{Vascular sarcoma}

Vascular sarcomas include both angiosarcoma and lymphangiosarcoma. Malignant vascular sarcomas comprise only approximately $2 \%$ of all soft tissue sarcomas in the adult population [49]. These highly malignant sarcomas are rare in the adult population and even rarer in the pediatric population. Lymph node metastases in vascular sarcomas range from $11 \%$ to $40 \%$ [25,28-30]. A more recent study notes regional lymphatic spread in $25 \%$ of patients with angiosarcoma [50]. However, the potential utility of SLN biopsy in the management of these tumors is less likely than with other sarcomas with a propensity to nodal spread.

Vascular sarcomas are notoriously difficult to achieve local control and often present with disseminated disease. In one study, 42 of 67 patients developed recurrent local disease after definitive local excision [49]. Over half of patients with angiosarcoma presented with metastatic disease on first admission, with 5-year survival rates of just over $20 \%[49,50]$. The role of radiation therapy and chemotherapy in the management of these tumors is uncertain and many authors report negligible benefits with either treatment modality. While it is feasible that SLN biopsy may have a role in targeting cases where radiation therapy or chemotherapy might have the most benefit, the inability to achieve local control and the frequency of metastatic disease at presentation make its potential utility difficult to support.

\section{Conclusion}

Sentinel lymph node (SLN) biopsy has revolutionized the management of melanoma and breast cancer, and is beginning to be applied to other tumors with a propensity for lymphatic spread. Although as a whole sarcomas do not have such a propensity, certain types of sarcomas have rates of nodal metastases comparable to that for an intermediate thickness cutaneous melanoma (10-20\%). The sentinel node biopsy procedure has a very low morbidity and a high likelihood of success, particularly for extremity primaries. As with melanoma and breast cancer, questions exist regarding the impact of selective regional lymph node dissection on distant relapse free survival and overall survival. In addition, the prognostic information obtained from nodal involvement of these sarcomas and the benefit of adjuvant systemic therapy in these patients is less clear than for other malignancies. In order to determine how best to use this technology in the rare types of sarcoma for which it may be ultimately useful, prospective multicenter clinical trials should be conducted. For the vast majority of soft tissue sarcomas, however, SLN biopsy does not have a role to play, and its indiscriminate use should be condemned.

\section{References}

[1] Cabanas RM. An approach to the treatment of penile carcinoma. Cancer 1977;39:456-66.

[2] Morton DL, Wen DR, Wong JH, et al. Technical details of intraoperative lymphatic mapping for early stage melanoma. Archives of Surgery 1992;127:392-9.

[3] Morton DL, Thompson JF, Essner R, et al. Validation of the accuracy of intraoperative lymphatic mapping and sentinel lymphadenectomy for early stage melanoma: a multicenter trial. Annals of Surgery 1999;230(4):453-65.

[4] Reintgen D, Cruse CW, Wells K, et al. The orderly progression of melanoma nodal metastases. Annals of Surgery 1994;220(6): 759-67.

[5] Gershenwald JE, Thompson W, Mansfield PF, et al. Multiinstitutional melanoma lymphatic mapping experience: the prognostic value of sentinel lymph node status in 612 stage I or II melanoma patients. The Journal of Clinical Oncology 1999;17(3): 976-83.

[6] Krag D, Weaver D, Ashikaga T, et al. The sentinel node in breast cancer: a multicenter validation study. The New England Journal of Medicine 1998;339(14):941-6.

[7] Chu KU, Turner RR, Hansen NM, et al. Sentinel node metastasis in patients with breast carcinoma accurately predicts immunohistochemically detectable nonsentinel node metastasis. Annals of Surgical Oncology 1999;6(8):756-61

[8] Sabel MS, Zhang P, Barnwell JM, Winston JS, Hurd TC, Edge SE. Accuracy of sentinel node biopsy in predicting nodal status in patients with breast carcinoma. Journal of Surgical Oncology 2001;77:243-6.

[9] Liptay MJ, Grondin SC, Fry WA, et al. Intraoperative sentinel lymph node mapping in non-small-cell lung cancer improves detection of micrometastases. The Journal of Clinical Oncology 2002;20(8):1984-8

[10] Bendavid Y, Latulippe JF, Younan RJ, et al. Phase I study on sentinel lymph node mapping in colon cancer: a preliminary report. Journal of Surgical Oncology 2002;79(Suppl. 2):81-4.

[11] Esser S, Reilly WT, Riley LB, Eyvazzadeh C, Arcona S. The role of sentinel lymph node mapping in staging of colon and rectal cancer. Diseases of the Colon and Rectum 2001;44(6):850-4

[12] Altinyoller H, Bergeroglu U, Celen O. Lymphatic mapping and sentinel lymph node biopsy in squamous cell carcinoma of the lower lip. European Journal of Surgical Oncology 2002;28(1): $72-4$.

[13] Wiseman SM, Loree TR, Hicks Jr WL, Rigual NR. Sentinel lymph node biopsy in SCC of the head and neck: a major advance in staging the N0 neck. Ear Nose \& Throat Journal 2002; 81(3): 156-60.

[14] Holub Z, Jabor A, Kliment L. Comparison of two procedures for sentinel lymph node detection in patients with endometrial cancer: a pilot study. European Journal of Gynaecological Oncology 2002;23(1):53-7.

[15] Levenback C, Coleman RL, Burke TW, Bodurka-Bevers D, Wolf JK, Gershenson DM. Intraoperative lymphatic mapping and sentinel node identification with blue dye in patients with vulvar cancer. Gynecologic Oncology 2001;83(2):276-81.

[16] Sliutz G, Reinthaller A, Lantzsh T, et al. Lymphatic mapping of sentinel nodes in early vulvar cancer. Gynecologic Oncology 2002;84(3):449-52.

[17] Fukui Y, Yamakawa T, Taniki T, Numoto S, Miki H, Monden Y. Sentinel lymph node biopsy in patients with papillary thyroid carcinoma. Cancer 2001;92(11):2868-74. 
[18] Gallowitsch HJ, Mikosch P, Kresnick E, Starlinger M, Lind P. Lymphoscintigraphy and gamma probe-guided surgery in papillary thyroid carcinoma. Clinical Nuclear Medicine 1999;24(10): 744-6.

[19] Mehrany K, Otley CC, Weenig RH, Phillips PK, Roenigk RK, Nguyen TH. A meta-analysis of the prognostic significance of sentinel lymph node status in merkel cell carcinoma. Dermatologic Surgery 2002;28(2):113-7.

[20] Coit DG. Merkel cell carcinoma. Annals of Surgical Oncology 2001;8(Suppl. 9):99S-102S.

[21] Allen PJ, Busam K, Hill AD, Stojadinovic A, Coit DG. Immunohistochemical analysis of sentinel lymph nodes from patients with Merkel cell carcinoma. Cancer 2001;92(6):1650-5.

[22] Aikou T, Higashi H, Natsugoe S, Hokita S, Baba M, Tako S. Can sentinel node navigation surgery reduce the extent of lymph node dissection in gastric cancer? Annals of Surgical Oncology 2001;8(Suppl. 9):82S-5S.

[23] Yasuda S, Shimada H, Ogoshi K, et al. Preliminary study for sentinel lymph node identification with Tc-99m tin colloid in patients with esophageal or gastric cancer. Tokai Journal of Experimental \& Clinical Medicine 2001;26(1):15-8.

[24] Liptay MJ, Grondin SC, Fry WA, et al. Intraoperative sentinel lymph node mapping in non-small-cell lung cancer improves detection of micrometastases. Journal of Clinical Oncology 2002;20:1984-8.

[25] Skinner KA, Eilber FR. Soft tissue sarcoma nodal metastases: biologic significance and therapeutic considerations. Surgical Oncology Clinics of North America 1996;5(1):121-7.

[26] Potter DA, Glenn J, Kinsella T, et al. Patterns of recurrence in patients with high grade soft tissue sarcomas. Journal of Clinical Oncology 1985;3:353-66.

[27] Vezeridis MP, Moore R, Karakousis CP. Metastatic patterns in soft tissue sarcomas. Archives of Surgery 1983;118:915-8.

[28] Fong Y, Coit DG, Woodruff JM, et al. Lymph node metastases from soft tissue sarcoma in adults. Analysis of data from a prospective database of 1772 sarcoma patients. Annals of Surgery 1993;217:72-7.

[29] Mazeron JJ, Suit HD. Lymph nodes as sites of metastases from sarcomas of soft tissue. Cancer 1987;60:1800-8.

[30] Weingrad DN, Rosenberg SA. Early lymphatic spread of osteogenic and soft-tissue sarcomas. Surgery 1978;84:231-40.

[31] Lawrence Jr W, Hays DM, Heyn R, Tefft M, Crist W, Beltangady M, Newton Jr W, Wharam M. Lymphatic metastases with childhood rhabdomyosarcoma A Report from the Intergroup Rhabdomyosarcoma Study. Cancer 1987;60(4):910-5.

[32] Neville HL, Andrassy RJ, Lobe TE, Bagwell CE, Anderson JR, Womer RB, Crist WM, Wiener ES. Preoperative staging, prognostic factors, and outcome for extremity rhabdomyosarcoma: a preliminary report from the intergroup rhabdomyosarcoma Study IV (1991-1997). Journal of Pediatric Surgery 2000; 35(2):317-21.

[33] Tefft M, Hays D, Raney RB, Lawrence W, Soule E, Donaldson $\mathrm{MH}$, Sutow WW, Gehan E. Radiation to regional nodes for rhabdomyosarcoma of the genitourinary tract in children: is it necessary? A Report from the Intergroup Rhabdomyosarcoma study no. 1 (IRS-1). Cancer 1980;45(12):3065-8.

[34] Wiener ES, Anderson JR, Ojimba JI, et al. Controversies in the management of paratesticular rhabdomyosarcoma: is staging retroperitoneal lymph node dissection necessary for adolescents with resected paratesticular rhabdomyosarcoma? Seminars in Pediatric Surgery 2001;10(3):146-52.

[35] Esnaola NF, Rubin BP, Baldini EH, Vasudevan N, Demetri GD, Fletcher CD, Singer S. Response to chemotherapy and predictors of survival in adult rhabdomyosarcoma. Annals of Surgery 2001;234(2):215-23.

[36] Spillane AJ, Thomas JM, Fisher C. Epithelioid sarcoma: the clinicopathological complexities of this rare soft tissue sarcoma. Annals of Surgical Oncology 2000;7(3):218-25.

[37] Chase DR, Enzinger FM. Epithelioid sarcoma: diagnosis, progostic indicators and treatment. American Journal of Surgical Pathology 1985;9:241-63.

[38] Ross HM, Lewis JJ, Woodruff JM, et al. Epithelioid sarcoma: clinical behavior and prognostic factors of survival. Annals of Surgical Oncology 1997;4:491-5.

[39] Spillane AJ, Thomas JM, Fisher C. Epithelioid sarcoma: the clinicopathological complexities of this rare soft tissue sarcoma. Annals of Surgical Oncology 2000;7(3):218-25.

[40] Callister MD, Ballo MT, Pisters PWT, et al. Epithelioid sarcoma: results of conservative surgery and radiotherapy. International Journal of Radiation Oncology Biology Physics 2001;51(2): 384-91.

[41] Halling AC, Wollan PC, Pritchard DJ, et al. Epithelioid sarcoma: a clinicopathologic review of 55 cases. Mayo Clinic Proceedings 1996;71:636-42.

[42] Whitworth PW, Pollock RE, Mansfield PF, et al. Extremity epithelioid; sarcoma amputation vs. local resection. Archives of Surgery 1991;126:1485-9.

[43] Wolchok JD, Wolchok J, Antonescu CR, et al. Clear cell sarcoma/malignant melanoma of soft parts (CCS/MMSP) is more closely related to melanoma than sarcoma, based on oligonucleotide array analysis. Proceedings of the American Society for Clinical Oncology 2002 (abstract).

[44] Finley JW, Hanypsiak B, McGrath B, Kraybill W, Gibbs JF. Clear cell sarcoma: the Roswell Park experience. Journal of Surgical Oncology 2001;77(Suppl. 1):16-20.

[45] Mark RJ, Poen JC, Tran LM, Fu YS, Juillard GF. Angiosarcoma. A Report of 67 patients and a Review of the Literature. Cancer 1996;77:2400-6.

[46] Spillane AJ, A'Hern R, Judson IR, et al. Synovial sarcoma: a clinicopathologic, staging, and prognostic assessment. Journal of Clinical Oncology 2000;18:3794-803.

[47] Andrassy RJ, Okcu MF, Despa S, Raney RB. Synovial sarcoma in children: surgical lessons from a single institution and review of the literature. Journal of the American College of Surgeons 2001;192(3):305-13.

[48] Spillane AJ, A'Hern R, Judson IR, Fisher C, Thomas JM. Synovial sarcoma: a clinicopathologic, staging, and prognostic assessment. Journal of Clinical Oncology 2000;18(22): 3794-803.

[49] Mark RJ, Poen JC, Tran LM, Fu YS, Juillard GF. Angiosarcoma. A report of 67 patients and a review of the literature. Cancer 1996;77(11):2400-6.

[50] Naka N, Ohsawa M, Tomita Y, Kanno H, Uchida A, Myoui A, Aozasa K. Prognostic factors in angiosarcoma: a multivariate analysis of 55 cases. Journal of Surgical Oncology 1996;61 (Suppl. 3):170-6. 


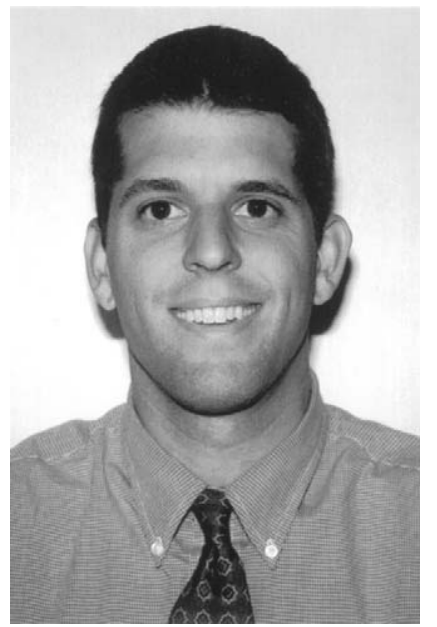

Dan G. Blazer III

Dan G. Blazer III is a general surgery resident at the University of Michigan, and is currently a research fellow in the laboratory of Dr. Steven Libutti in the Surgery Branch of the National Cancer Institute. The laboratory is principally involved in studying tumor angiogenesis in order to develop novel treatment strategies for human malignancies. Previous research efforts by Dr. Blazer have included the study of polymorphisms as markers for prostate cancer risk while a predoctoral fellow at the National Institute of Environmental Health Sciences and the investigation of novel gene and immune based therapies using preclinical models of carcinoma while a research assistant in the laboratory of Dr. H. Kim Lyerly at Duke University. He intends to pursue a surgical oncology fellowship upon the completion of his surgical residency.

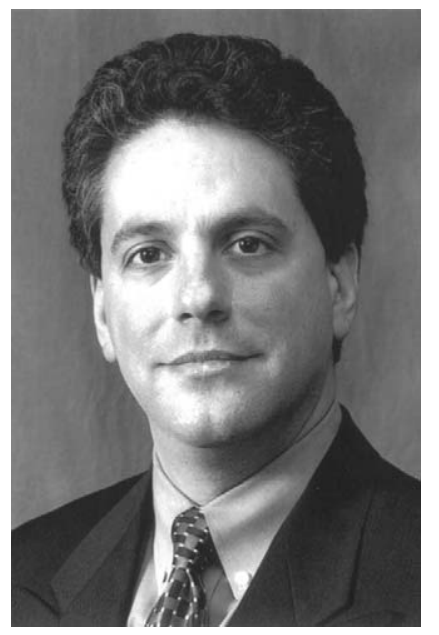

\section{Michael S. Sabel}

Michael S. Sabel, M.D. is Assistant Professor of Surgery in the Division of Surgical Oncology at the University of Michigan, Comprehensive Cancer Center. Trained initially at Rush-Presbyterian-St. Luke's Medical Center in Chicago, he completed an NIH-T32 fellowship in tumor immunology and surgical oncology fellowship at Roswell Park Cancer Institute in Buffalo, NY. Dr.
Sabel's clinical interests are in melanoma, sarcoma and breast cancer. His laboratory interests focus on neoadjuvant immunotherapy; the generation of a systemic immune response to a tumor in situ, prior to surgical excision. To date, much of his research has centered on the intratumoral delivery of IL-12, GM-CSF and/or $\mathrm{TNF}-\alpha$ and the immunologic response to cryoablation.

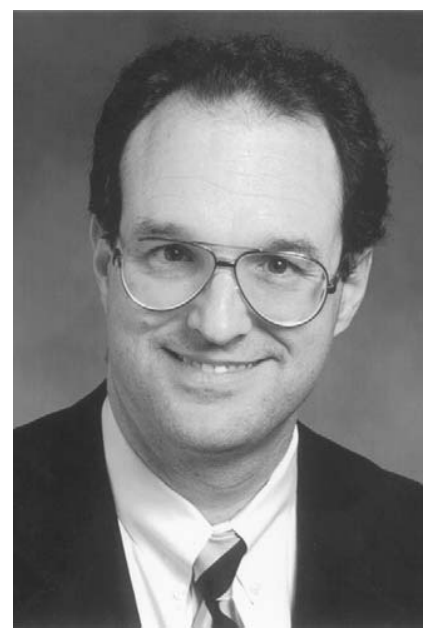

Vernon K. Sondak

Vernon K. Sondak, M.D. received his undergraduate and M.D. degrees from Boston University, prior to residency training at the University of California, Los Angeles. In 1987, he joined the faculty at the University of Michigan Medical Center, Ann Arbor, MI. He is currently Professor of Surgery in the Division of Surgical Oncology. Dr. Sondak specializes in the treatment of soft tissue tumors, including melanomas and sarcomas, and has had a long-standing interest in the surgical staging of regional lymph nodes by sentinel lymph node biopsy. 